\title{
Looking critically at the paradigm of radiation exposure from multiple imaging examinations
}

\author{
Donald P. Frush ${ }^{1}$ (D) - Guy Frija ${ }^{2}$
}

Received: 30 November 2021 / Revised: 9 January 2022 / Accepted: 12 January 2022 / Published online: 3 February 2022

(C) The Author(s), under exclusive licence to European Society of Radiology 2022

The value of justified medical imaging is unquestioned, whether as an isolated occurrence or recurrent. For recurrent examinations, "multiple imaging examinations" (MIEs) can be defined as any radiological imaging examination that occurs subsequent to an initial examination. This can be in several scenarios: necessary or reasonable follow-up imaging based on results of the initial examination, evaluation based on unanticipated clinical course related or unrelated to that resulting in the initial exam, standard follow-up for oncological or other chronic condition, or screening. The paradigm of MIEs and the resultant measure of cumulative effective dose (CED) is drawing increased scrutiny $[1,2]$.

MIEs are a normal occurrence as the information is crucial for treatment management. A single examination may be even an exception in some centers. MIEs are often prescribed by protocol. In oncology, imaging history provides essential biomarkers for evaluating the treatment response and where the potential for resultant stochastic effects is likely lower than would occur in the general population given the often disease-related reduced life-expectancy. In two recent surveys, oncology represented $80-90 \%$ of the settings for MIEs $[3,4]$.

Attention has been drawn recently to the risks induced by MIEs in particular when the CED is $\geq 100 \mathrm{mSv}$ [1]. The principles of medical radiation protection are that unnecessary examinations should be avoided and radiation exposure for any single examination, including those resulting in cumulative exposures, should be as low as reasonably achievable. Labeling of any group at or beyond a $\mathrm{mSv}$ threshold as needing additional consideration implies that decisions adhering to

Donald P. Frush

donald.frush@duke.edu

1 Duke University Medical Center, Box 3808, Durham, NC 27710, USA

2 Université de Paris, Paris, France the principles of radiation protection along the way by the medical community have in some measure not been adhered to or are insufficient. Any CED then may be thought of as a necessary result of informed decision-making embracing the familiar principles of radiation protection for each examination. Decisions based on cumulative effective dose, and attendant real or potential small biological risks should also consider the far greater cumulative benefit. The influence of the regional practice on CED should also be considered as there are radiation exposure differences for the same clinical context $[5,6]$ making a fixed CED reference value challenging. The CED paradigm suffers too from the significance of regional anatomic risks from the same 100-mSv "alert", such as from recurrent brain versus abdomen pelvis $\mathrm{CT}$ examinations. Finally, the emphasis on thresholds for CED in decisions for subsequent imaging may also create a level of concern that could result in referrers, patients, or their caregivers resisting or refusing otherwise indicated examinations. Emphasis on cumulative dose may impart cumulative anxiety.

There are other challenges with global adherence to a paradigm of CED. Age and other patient characteristics for life expectancy also should be taken into consideration when assessing the relevance of CED and potential efforts in advancing management. High CED for oncologic and nononcologic MIEs is also much less frequent in the pediatric population [3, 7]. Reports on the frequency and levels of CED for any clinical scenario can also be outdated. In nononcological diseases, the replacement of ionizing radiation modalities by MR imaging and ultrasound is increasing in imaging strategies.

Current dose management system (DMS) models can provide information for each patient on the number and type of examinations and on the effective dose for each examination over the course of the patient's medical care [8]. The penetration of these systems though is not universal and the issue is how this information should be curated. A high number of examinations in the patient history should not preclude the performance of a supplementary imaging test provided it is 
clinically justified $[2,9]$. This also follows since there is no consensus that a high CED represents a clinically relevant threshold. DMSs could provide alert levels, but disorderspecific thresholds are far from being defined. For example, oncological patients very often require a substantial number of follow-up radiological examinations and procedures leading to an appropriate CED, even those that may be considered large. Appropriate surveillance or other interim evaluation (i.e., unexpected change in clinical status) of patients with chronic conditions may also lead to appropriate, relatively high cumulative radiation exposures obviating the need to react to such exposures.

Responsibility for the monitoring and establishing what if any amount constitutes a high CED from MEIs should be shared. MEI pathways, both using ionizing and nonionizing radiation modalities, should be established through decisionmaking with referring service lines (including mindful design for industry trials) that include guidance from previous imaging (e.g., potential for a follow-up lower dose CT examination). These partnerships with clinical specialists can also help identify what, if any, representation of individual patient exposure record (i.e., number and type of examination, CED, or potentially individual organ dose) would be most valuable for that particular medical specialty for the various scenarios that might encompass MIEs. Inherent in this partnership is the availability to providers of applicable MIE information through frameworks such as clinical decision support.

The application of the CED paradigm for point-of-care imaging decisions should not be based on either CED alone or as a factor $[2,9]$. Imaging decisions are to be based on a benefit-risk approach embedded in adherence to the existing principles of justification based on clinical needs, attending as always to optimization. For all individuals undergoing MIEs, existing provisions such as the Bonn Call for Action [10] should be adhered to by the medical community and are equally applicable to single and multiple examination scenarios. We should continue to advance our ability to be accountable for the dose estimates for what we do, most effectively through DMS, which include measures (such as CED) that afford relevant representation of radiation exposures from MIEs. Responsibility also consists of educational efforts on CED metrics, including shortcomings of a single or cumulating effective dose, and identification of clinical situations where CED can be understood and defended by partnerships between imaging experts and clinicians to provide quality and safe care over time.

Acknowledgements The content of this manuscript was reviewed and approved by the International Society of Radiology Executive Committee.

Funding The authors state that this work has not received any funding.

\section{Declarations}

Guarantor The scientific guarantor of this publication is Donald P. Frush.

Conflict of interest The authors of this manuscript declare no relationships with any companies, whose products or services may be related to the subject matter of the article.

Statistics and biometry No complex statistical methods were necessary for this editorial.

Informed consent Not applicable for this editorial.

Ethical approval Institutional Review Board approval was not required because this is an editorial.

\section{References}

1. Brambilla M, Vassileva J, Kuchcinska A, Rehani MM (2020) Multinational data on cumulative radiation exposure of patients from recurrent radiological procedures: call for action. Eur Radiol 30:2493-2501

2. AAPM/ACR/HPS Joint Statement on Proper Use of Radiation Dose Metric Tracking for Patients Undergoing Medical Imaging Exams 08.06.21. Available: https://www.aapm.org/org/policies/ details.asp?id=1533 [Last accessed January 4, 2022]

3. Rehani MM, Yang K, Melick ER et al (2019) Patients undergoing recurrent CT scans: assessing the magnitude. Eur Radiol 450:18281836

4. Frija G, Damilakis J, Paulo G et al (2021) Cumulative effective dose from recurrent CT examinations in Europe: proposal for clinical guidance based on an ESR EuroSafe Imaging survey. Eur Radiol 31:5514-5523

5. Tsapaki V, Damilakis J, Paulo G et al (2021) CT diagnostic reference levels based on clinical indications: results of a large-scale European survey. Eur Radiol 31:4459-4469

6. Kanal KM, Butler PF, Sengupta D et al (2017) U.S. diagnostic reference levels and achievable doses for 10 adult $\mathrm{CT}$ examinations. Radiology 284:120-133

7. Frush D (2021) The cumulative radiation dose paradigm in pediatric imaging. Br J Radiol 94:epub ahead of print. https://doi.org/10. 1259/bjr.20210478

8. Loose RW, Vano E, Mildenberger P et al (2020) Dose management- requirements and recommendations for users. EuroSafe Imaging:ESI-11078. https://doi.org/10.26044/esi2020/ ESI-11078 [Last accessed January 4, 2022]

9. Sodickson AD (2021) Radiation concerns in frequent flyer patients: should imaging history influence decisions about recurrent imaging? Br J Radiol 94:epub ahead of print. https://doi.org/10.1259/bjr. 20210543

10. International Atomic Energy Agency, "Bonn Call for Action platform," 2017. Available: https://www.iaea.org/resources/rpop/ resources/bonn-call-for-action-platform [Last accessed January 4, 2022]

Publisher's note Springer Nature remains neutral with regard to jurisdictional claims in published maps and institutional affiliations. 